\title{
Editorial
}

\section{Orthopaedics and the COVID-19 pandemic}

\author{
Prannoy Paul* \\ Department of Orthopedics, Government Medical College, Kannur, Kerala, India
}

Received: 24 September 2020

Accepted: 07 November 2020

\author{
*Correspondence: \\ Dr. Prannoy Paul, \\ E-mail: prannoypaul@gmail.com
}

Copyright: () the author(s), publisher and licensee Medip Academy. This is an open-access article distributed under the terms of the Creative Commons Attribution Non-Commercial License, which permits unrestricted non-commercial use, distribution, and reproduction in any medium, provided the original work is properly cited.

The ongoing COVID-19 pandemic has affected healthcare systems across the world in an unprecedented manner. Several healthcare providers are now being recruited for COVID management from their original departments. In addition, reallocation of the available resources and the current economic confinements have forced hospitals worldwide to be restructured in a way so as to provide the best possible care for the patients, while preventing the transmission of coronavirus among patients as well as among healthcare providers.

The daily practice in orthopaedics has been tremendously affected due to the ongoing pandemic, just like any other medical field. Several changes have to be made in the management of inpatients, restricting the visiting hours in the out-patient departments, revised patient isolation policies and postponement of many elective surgeries. Many elective procedures, which constitute a considerable part of all orthopaedic procedures, now have to be postponed to reallocate healthcare personnel and resources like personal protective equipment and intensive care units (ICUs) for management of the COVID-19 crisis. At the same time, several conditions like severe trauma, infections or certain tumours would need urgent attention and management. This is therefore slowly restructuring the orthopaedic routine in hospitals across the world.

It is now known that the major routes of COVID-19 infection is through aerosols and through contaminated surfaces. ${ }^{1}$ In addition, aerosol generating procedures (AGPs) such as cardiopulmonary resuscitation, tracheal intubation, bronchoscopy, tracheostomy and non-invasive ventilation can produce airborne particles that are highly virulent and infectious. ${ }^{2}$ Though majority of the virus transmission occurs from symptomatic patients, a large number of asymptomatic patients can also be infective and should never be neglected. ${ }^{3}$ The virus is known to survive up to 3 hours in aerosols, 24 hours on cardboard, 2 days on clothes, 3 days on plastic and metal surfaces, 4 days on currency notes and up to a week on a used infected surgical mask. ${ }^{4}$ Therefore proper ventilation of closed environments, frequent sanitizing of hands, decontamination of surfaces, proper disposal of infective wastes and wearing mask is mandatory to avoid the infection.

Several types of masks are now available with different efficiencies in filtration. Normal surgical masks are not proven to be protective against droplets when tested under laboratory conditions. ${ }^{4}$ However, during non-aerosol generating procedures (AGPs), surgical masks may provide protection similar to an N95 respirator according to recent evidences..$^{5}$ Thus, whenever an N95 respirator is not available, healthcare personnel shall be encouraged to wear a surgical mask. Respirators are classified based on the percentage of particles $\geq 300 \mathrm{~nm}$ that it can effectively filter out. Thus an N95 mask can filter out $95 \%$ of particles $\geq 300 \mathrm{~nm}$. According to European standards, these respirators are classified as FFP1, FFP2 and FFP3, with their filtering capacities being $\geq 84 \%, \geq 94 \%$ and $\geq 99 \%$ respectively. The World Health Organization (WHO) recommends that all healthcare personnel should wear a respirator $(\geq \mathrm{N} 95$ or $\geq \mathrm{FFP} 2)$ during aerosol generating procedures, and in all other times, use at least a surgical mask, if a respirator is not available. ${ }^{6}$

The socio-economic and lifestyle changes created due to the pandemic has somewhat changed the diversity of cases presenting to the orthopaedic departments. Lockdown, quarantine and encouragement of work-from-home has led 
to a decrease in vehicle accidents and many occupational injuries. Closure of schools, however have resulted in a slight increase in the numbers of paediatric trauma cases. More elderly people are likely to be at home with the absence of caregivers, pointing to a foreseeable increase in geriatric fractures.

The pandemic has resulted in redefining the indications for several elective procedures while at the same time giving serious attention to the emergency interventions required, especially for those who are infected or suspected with COVID 19. The American academy of orthopaedic surgeons (AAOS) and the American college of surgeons (ACS) recommend judicious postponing of all elective surgeries considering the available resources and the prevalence of coronavirus in that particular region. ${ }^{7,8}$ Continuation of routine procedures as usual may result in a dangerous shortage of resources and manpower in case of an unexpected turn of events which may occur anytime.

A procedure is generally considered to be elective when no long term or short term complications are likely to occur if the procedure is delayed. However the reported pain and disability can be patient subjective and can create confusions in the decision making process by the surgeon. The Ohio hospital association recommends to defer the procedures that do not fall into the following category: "threat to the patient's life if surgery or procedure is not performed, threat of permanent dysfunction of an extremity or organ system, risk of metastasis or progression of staging, risk of rapidly worsening to severe symptoms". 9 The centres for medicare and medicaid services (CMS) propose a 3 tier system, to classify the urgency of the surgical procedure. ${ }^{10}$ They recommend postponing tier 1a operations like carpal tunnel release, to consider deferring tier 2 a surgeries like joint replacements and continue with tier 3 a surgeries like severe trauma and patients how are very symptomatic. From an extensive review of the current guidelines and recommendations, Luca et al enlists the orthopaedic conditions that require urgent management as follows: compartment syndrome, open fractures, periprosthetic fractures, joint dislocations, acute limb ischemia (including traumatic amputations), septic arthritis, cauda equina syndrome, hip and intertrochanteric fractures in the elderly, pelvic fractures, spinal cord compression or radiculopathy due to unstable vertebral fractures, spinal tumor, disc herniation, epidural abscess or hematoma with worsening neurological symptoms and/or intractable pain, complex fractures or long bone fractures that may lead to loss of function or permanent disability if treated non-operatively, and necrotizing fasciitis. ${ }^{11}$

Even if the COVID situation in a region is in control so as to allow for elective procedures, it is advisable to follow an early discharge policy, as studies have shown that hospitalisation under 24 hours reduces the risk of transmission of infection as well as does not significantly affect the available health resources. ${ }^{12}$ Also, from his retrospective study on patients with COVID-19 and bone fractures $\mathrm{Mi}$ et al reported an increased morbidity and mortality in patients treated by open reduction and internal fixation. Hence, non-operative treatment shall be preferred in cases where it is applicable, especially in the elderly. ${ }^{13}$

Same day admission is preferred for all surgical cases, and upon arrival, each patient and caregiver shall be assessed for COVID-19 risk factors, checked for temperature and shall be made to wear a surgical mask at all times. COVID19 testing of the patient using real time polymerase chain reaction (RT-PCR) is recommended depending on the resources available in the region. ${ }^{14}$

Operating rooms are usually closed spaces and are usually maintained at positive pressures to reduce surgical contamination. This can however, result in transmission of the virus. Conversion of the operating rooms to negative pressure systems are ideal, but if not feasible, turning off the positive pressure system and adding a portable highefficiency particulate air (HEPA) filtration system would be effective. ${ }^{15}$ Minimum number of staff shall be present in the operating room and unnecessary entry and exit of staff from the operating rooms shall be discouraged. All staff are required to wear adequate personal protective equipment with respirators (FFP2/N95 are recommended) instead of regular surgical masks in the operating rooms. When possible, prone positioning of the patient can reduce the transmission through respiratory droplets. The surgical approach requiring the least operating time shall be preferred. ${ }^{15}$ Avoiding high speed drills/saw can reduce the generation of aerosols. Electrocautery shall be minimised and used only with a suction device. Absorbable sutures shall be preferred to decrease the number of post-operative visits. Transparent wound dressings can enable remote wound inspection. Splints instead of a plaster of can also help for similar reasons. ${ }^{15}$

Compartmentalisation of department activity by dividing the staff into different pools are shown to be effective in preventing an outbreak within a department. The orthopaedic department may be divided into 3 pools, an inpatient pool dealing with the admitted patients, on call services and interdepartmental consultations, an outpatient pool to attend to the unavoidable patient visits in the outpatient department (OPD), and a surgical pool, for the unavoidable operative procedures. Each pool can rotate every one to two weeks with a period of isolation for 14 days. Each pool shall have different workstations and members of different pools are not supposed to not be in contact with each other to prevent cross contamination. ${ }^{16}$ Also the elder faculty and the ones with serious comorbidities shall be in the pools with minimal risk of getting an infection.

Outpatient clinics should follow all the necessary safety precautions mentioned earlier, and tele-medicine consultations over video call based platforms can be used whenever possible. Tele-medicine saves precious medical resources like personal protective equipment while enabling regular follow up. 
The pandemic has also affected the regular academic sessions and the continuous process of acquiring clinical skills for the orthopaedic resident students. These residents are the future of our profession and hence their training cannot be neglected. The resident program directors shall ensure that the resident training is not interrupted due to the pandemic, by making use of modern leaning tools like simulations and virtual classrooms. While academic sessions can be continued through online video conferencing platforms, clinical and surgical training may be continued through cadaveric dissection, simulators and virtual reality tools. ${ }^{17}$

Due to various constraints caused due to this pandemic, often physicians may not be able to provide their patients with the best care that was routinely being provided. This may cause ethical or moral dilemmas for many physicians. But amid this pandemic physicians need to change from a strictly patient centered management to a more community oriented view. For instance conservative management shall be preferred for many conditions whenever possible. Ensuring safety of the general population and the healthcare providers is required to provide uninterrupted care for patients requiring urgent attention.

As the dynamics of the pandemic is changing, we have to be flexible and prompt in making necessary changes in the system to provide uninterrupted care for those in need while not compromising on safety of the healthcare personnel and of the general population. This pandemic has changed departmental routines in ways we have never imagined. But this fight against our common enemy is awakening a sense of fraternity among the scientific community like never before. Let us hope we win this war soon without suffering much loses.

\section{REFERENCES}

1. Ashford RU, Nichols JS, Mangwani J. Annotation: the COVID-19 pandemic and clinical orthopaedic and trauma surgery. J Clin Orthop Trauma. 2020;11(3):504-5.

2. WHO. Infection prevention and control during healthcare when COVID-19 is suspected. 2020. Available at: https://www.who.int/publicationsdetail/infection-prevention-and-control-duringhealth-care-when-novel-coronavirus-(ncov)infection-is-suspected-20200125. Accessed on: 25 August 2020.

3. Bai Y, Yao L, Wei T, Tian F, Jin DY, Chen L, et al. Presumed asymptomatic carrier transmission of COVID-19. JAMA. 2020;323(14):1406-7.

4. Viswanath A, Monga P. Working through the COVID-19 outbreak: rapid review and recommendations for MSK and allied heath personnel. J Clin Orthop Trauma. 2020;11(3):500-3.

5. Bartoszko JJ, Farooqi MAM, Alhazzani W, Loeb M (2020) Medical masks vs N95 respirators for preventing COVID-19 in health care workers a systematic review and meta-analysis of randomized trials. Influenza Other Respir Viruses. 2020;14(4):365-73.

6. WHO. Advice on the use of masks in the context of COVID-19. 2020. Available at: https://www.who.int/publications-detail/advice-onthe-use-of-masks-in-the-community-during-homecare-and-in-healthcare-settings-in-the-context-ofthe-novel-coronavirus-(2019-ncov)-outbreak.

Accessed on: 25 August 2020.

7. Guy DK, Bosco JA, Savoie FH. AAOS Guidelines for Elective Surgery During the COVID-19 Pandemic. 2020. Available at: https://www.aaos.org/about/covid-19-informationfor-our-members/aaos-guidelines-for-electivesurgery. Accessed on: 25 August 2020.

8. ACS. COVID-19: Recommendations for Management of Elective Surgical Procedures. 2020. Available at: https://www.facs.org/covid-19/clinicalguidance/elective-surgery. Accessed on: 25 August 2020.

9. Sarac NJ, Sarac BA, Schoenbrunner AR, Janis JE, Harrison RK, Phieffer LS, et al (2020) A review of state guidelines for Elective Orthopaedic procedures during the COVID-19 outbreak. J Bone Joint Surg Am. 2020;102(11):942-5.

10. CMS. Non-Emergent, Elective Medical Services, and Treatment Recommendations. 2020. Available at: https://www.cms.gov/files/document/cms-nonemergent-elective-medical-recommendations.pdf. Accessed on: 25 August 2020.

11. Ambrosio L, Vadalà $\mathrm{G}$, Russo $\mathrm{F}$, Papalia $\mathrm{R}$, Denaro $\mathrm{V}$, et al. The role of the orthopaedic surgeon in the COVID-19 era: cautions and perspectives. J Exp Ortop. 2020;7:35.

12. Chang Liang Z, Wang W, Murphy D, Po Hui JH. Novel Coronavirus and Orthopaedic Surgery: Early Experiences from Singapore. J Bone Joint Surg Am. 2020;102(9):745-9.

13. Mi B, Chen L, Xiong Y, Xue H, Zhou W, Liu G. Characteristics and early prognosis of COVID-19 infection in fracture patients. J Bone Joint Surg Am. 2020;102(9):750-8.

14. WHO. Global surveillance for COVID-19 caused by human infection with COVID-19 virus. 2020. Available at: https://www.who.int/docs/defaultsource/coronaviruse/global-surveillance-for-covidv-19-final200321-rev.pdf. Accessed on: 25 August 2020.

15. Rodrigues-Pinto R, Sousa R, Oliveira A. Preparing to perform trauma and Orthopaedic surgery on patients with COVID-19. J Bone Joint Surg Am. 2020;102(11):946-50.

16. Massey PA, McClary K, Zhang AS, Savoie FH, Barton RS. Orthopaedic surgical selection and inpatient paradigms during the coronavirus COVID19 pandemic. J Am Acad Orthop Surg. 2020;28(11):436-50. 
17. Holland JP, Waugh L, Horgan A, Paleri V, Deehan DJ. Cadaveric hands-on training for surgical specialties: is this back to the future for surgical skills development? J Surg Educ. 2011;68:110-6.
Cite this article as: Paul P. Orthopaedics and the COVID-19 pandemic. Int J Res Orthop 2021;7:17982. 